\title{
An Improved Symbiosis Particle Swarm Optimization for Solving Economic Load Dispatch Problem
}

\author{
Jianxia Zhang $\left(\mathbb{D},{ }^{1}\right.$ Jianxin Zhang $\left(\mathbb{D},{ }^{2}\right.$ Feng Zhang $\left(\mathbb{D},{ }^{1}\right.$ Minglu Chi $\mathbb{D},{ }^{1}$ and Linbin Wan $\mathbb{D}^{3}$ \\ ${ }^{1}$ School of Intelligent Engineering, Henan Institute of Technology, Xinxiang 453003, China \\ ${ }^{2}$ School of Computer Science and Engineering, Dalian Minzu University, Dalian 116600, China \\ ${ }^{3}$ Student Affairs Office, Henan Institute of Technology, Xinxiang 453003, China \\ Correspondence should be addressed to Jianxia Zhang; zhangjianxia@hait.edu.cn and Jianxin Zhang; jxzhang0411@163.com
}

Received 6 September 2020; Revised 14 December 2020; Accepted 22 December 2020; Published 5 January 2021

Academic Editor: François Vallée

Copyright (c) 2021 Jianxia Zhang et al. This is an open access article distributed under the Creative Commons Attribution License, which permits unrestricted use, distribution, and reproduction in any medium, provided the original work is properly cited.

To realize the sustainable development of social economy, energy conservation and emission reduction has become one of the problems that must be considered in the current power system. Under the electric power market system, the economic load dispatch problem not only is important but also has practical significance and broad application prospects. In order to minimize the costs of electric-power generation, the load capacity should be reasonably assigned among many different generating sets. In this paper, an improved symbiosis particle swarm optimization algorithm was proposed, aiming at providing a better solution to this problem. First of all, a mathematical model was established with certain constraints, which successfully converted the practical problem into a mathematical one. Then, to balance the global optimization and local search capability, an improved symbiosis particle swarm optimization algorithm with mutualistic symbiosis strategy in nature was presented. The improved symbiosis particle swarm optimization algorithm consisted of three swarms inspired by the proverb "two heads are better than one," and its specific analysis was through the standard test functions. At last, the economic load dispatch problem could be optimized by the proposed improved symbiosis particle swarm optimization algorithm. In addition, two different kinds of practical examples were also adopted for algorithm evaluation. From the simulation results, it can be seen clearly that the costs of electric-power generation gained were the lowest compared with the results of particle swarm optimization algorithm, improved chaos particle swarm optimization algorithm, and symbiotic organisms search algorithm, well demonstrating the effectiveness of the improved symbiosis particle swarm optimization algorithm in solving the economic load dispatch problem.

\section{Introduction}

With the rapid development of market system and the establishment of resource-saving society, the economic load dispatch (ELD) has become an important and practical optimization problem [1-3]. It can be described as the following definition. It refers to the fact that the generator cost of the whole power system increases least, which is under the different constraint conditions [4]. The different constraint conditions include satisfying the user electric requirement, reducing the loss of power system, being in the set range of active power generation, and so on [5]. At present, the ELD problem affects the safety and economic benefit of the whole power system. How to reduce the generator cost of the whole power system has become the wide focus of attention of domestic and foreign scholars [6-8].

The traditional approaches for solving the ELD problem include the Lagrange method [9], the equal incremental principle [10], the sequential method [11], the point-bypoint method [12], and so on. These traditional approaches cannot solve the ELD problem well, because this problem has characteristics of very strong non-linearity and different various results. In recent years, with the constant development and extending application of intelligent optimization algorithms, how to solve the ELD problem using the intelligent optimization algorithms has become a hot topic for study [13]. In the year of 2011, a differential artificial bee colony algorithm on solving the ELD problem was proposed 
by Xiong et al. which was inspired by differential evolution algorithm. This algorithm not only improved the search behaviors of employed bees, but also added an effective repair procedure [14]. An improved chaos particle swarm optimization (ICPSO) algorithm had been presented by Han et al. to solve the ELD problem for thermal power plants in 2015. The ICPSO algorithm was based on the chaos particle swarm optimization (CPSO) algorithm. In order to increase the diversity of particles in the swarm, the crossover operation in genetic algorithm was adopted and the generation of inertia weight used the parabola and chaotic sequence [15]. In 2016, a novel hybrid algorithm of differential evolution (DE) and particle swarm optimization (PSO) for solving the ELD problem was described by Parouha and Das. It was abbreviated as "DPD," because it used DE-PSO-DE on a population composed of three swarms. It has better convergence and efficiency [16]. Together, a new approach to economic dispatch problem using a hybrid algorithm named ACO-ABC-HS was put forward by Sen and Mathur in the year of 2016, which combined the framework of ant colony optimization (ACO), artificial bee colony $(\mathrm{ABC})$, and harmonic search (HS). It could find out the optimum results satisfying minimizing the costs of generation [17]. In 2019, a new phasor particle swarm optimization (PPSO) algorithm was put forward by Gholamghasemi et al. to solve the nonconvex economic load dispatch problems. It is efficient and reliable [18]. In 2020, a Marine Predators Algorithm (MPA) was presented by Faramarzi et al., which has got better solutions with low computational cost compared with other algorithms [19]. An improved equilibrium optimization algorithm (IEOA) was described by Shaheen et al. in 2020. It can be used to optimize the multi-objective function of total active power loss minimization [20]. Besides, a multiobjective salp swarm optimizer (MSSO) was presented to enhance the performance of distribution systems. It was more advantageous in large-scale 118-node radial system [21].

In this paper, an improved symbiosis particle swarm optimization (ISPSO) algorithm was proposed. It mainly focused on solving the ELD problem. It is based on the principle of mutualistic symbiosis in nature. The mutualistic symbiosis refers to the fact that the biological populations can gain benefits and have mutual influence on each other. The ISPSO algorithm consists of three swarms, which was inspired by the proverb "two heads are better than one." The two swarms carried on iteration optimization through the PSO of linear descending inertia weight. The third swarm used the position information of the above two swarms to self-update. The specific remaining structure of this paper is organized as follows. In the second part, a mathematical model of ELD problem was established with certain constraint conditions. The concrete ISPSO algorithm was explained and analyzed in the third part. In the fourth part, the two different practical examples were used to do the experiments, which were compared with the PSO algorithm, the ICPSO algorithm, and the symbiotic organisms search (SOS) algorithm. Lastly, the conclusion is presented.

\section{Establishing a Mathematical Model of Economic Load Dispatch Problem}

The ELD problem can be represented as a nonlinear programming subject with inequality constraints and boundary constraints. With satisfying the several different conditions, the minimizing value of fitness function should be found out.

2.1. Different Constraint Conditions. The constraint conditions have two forms that include equality and inequality constraints. In this paper, the equality constraints refer to the fact that the obtained parameter values satisfy the balance of load power. The particular expression is as shown in the following formula:

$$
\sum_{i=1}^{n} P_{i}=P,
$$

where $n$ is the total number of generators, $P_{i}$ represents the active power of the $i$ th generator, and $P$ is the total load power of whole system.

In this paper, the inequality constraints mean that the active power of unit is less than or equal to the upper limit but greater than or equal to the lower limit, as is shown in the following formula:

$$
P_{i_{-} \min } \leq P_{i} \leq P_{i_{-} \max }, \quad i=1,2, \ldots, n,
$$

where $P_{i_{-} \text {min }}$ is the minimizing active power of the $i$ th generator and $P_{i_{-} \max }$ represents the maximizing active power of the ith generator.

2.2. Description of the Fitness Function. The fitness function in this paper is established with satisfying constraints (1) and (2). Furthermore, the valve-point effect of practical generator is also considered. The valve-point effect is that there will be a kind of phenomenon, which is named the wire drawing. It appears when the turbine inlet valve suddenly opened, which produces a pulse on the consumption characteristic curve of generator [22]. It can be expressed as the following formula:

$$
E_{i}=\left|e_{i} \sin \left[f_{i}\left(P_{i}-P_{i_{-} \min }\right)\right]\right|,
$$

where $e_{i}$ and $f_{i}$ both express the consumption characteristic parameters, which are the constants.

The consumption characteristic curve of generator can be expressed using the quadratic functions of active power, which is as shown in the following formula:

$$
F\left(P_{i}\right)=a_{i} P_{i}^{2}+b_{i} P_{i}+c_{i}
$$

where $a_{i}, b_{i}$, and $c_{i}$ are constants.

Considering the above various factors, the fitness function of ELD problem can be expressed as the following formula: 


$$
\min \text { fitness }\left(P_{i}\right)=\sum_{i=1}^{n}\left(a_{i} P_{i}^{2}+b_{i} P_{i}+c_{i}+\left|e_{i} \sin \left[f_{i}\left(P_{i}-P_{i \_ \text {min }}\right)\right]\right|\right) .
$$

\section{An Improved Symbiosis Particle Swarm Optimization Algorithm}

3.1. Overview of Particle Swarm Optimization. The PSO algorithm was studied by Drs. Eberhart and Kennedy in 1995 [23]. It was originated from the research of predatory behavior of birds, which is similar to other optimal algorithms based on swarm intelligence. The PSO algorithm has less parameters and better structure. Nor is this all; its iterative rules are relatively simple. It has a good application and promotion in various fields. In the PSO algorithm, there are two different variables that are velocity and position. Each

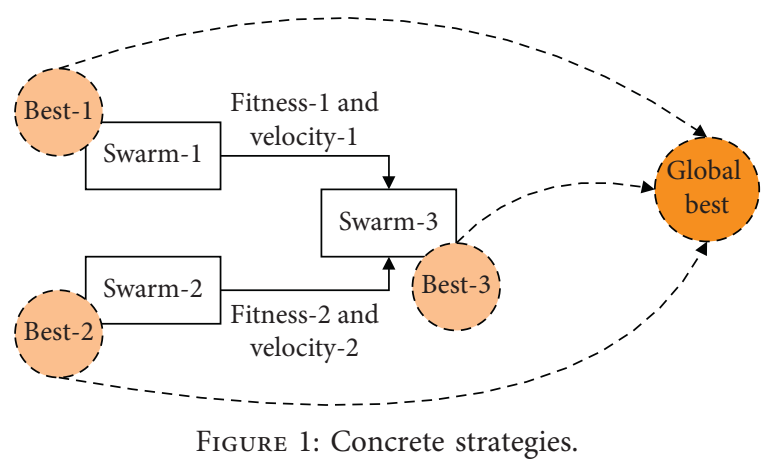

particle can update its own velocity and position through the optimal position of itself and overall situation. The expression in detail is as shown in the following formula:

$$
\left\{v_{i, j}(t+1)=w v_{i, j}(t)+c_{1} r_{1}\left[p_{i, j}-x_{i, j}(t)\right]+c_{2} r_{2}\left[p_{g, j}-x_{i, j}(t)\right] x_{i, j}(t+1)=x_{i, j}(t)+v_{i, j}(t+1),\right.
$$

where $v_{i, j}$ represents the velocity of the $i$ th particle, $j=1,2, \ldots, d, d$ is the dimension of searching space, $x_{i, j}$ expresses the position of the $i$ th particle. $t$ is the current iteration, $w$ indicates the inertia weight, $c_{1}$ and $c_{2}$ are the learning factors, $r_{1}$ and $r_{2}$ show the random number uniformly distributed between 0 and $1, p_{i, j}$ expresses the current optimal position of a particle, and $p_{g, j}$ represents the global optimal position.

\subsection{Description of Improved Symbiosis Particle Swarm Op-} timization Algorithm. The PSO algorithm has an advantage of simple easy operation. However, like other algorithms, it also has the inevitable disadvantage, such as easily trapping in local optimum. The SOS algorithm has slow convergence speed in the later stage. In order to improve the performance when the PSO algorithm and SOS algorithm solve complex problems, an ISPSO algorithm is proposed in this paper. The ISPSO algorithm is based on the strategy of mutualistic symbiosis in nature, which makes the particles support each other during the whole iterative process. What is more, the strategy is advantageous to creeping towards the optimal individual. It can make the whole swarm prevent the phenomenon of evolutionary stagnation. In this paper, inspired by the proverb "two heads are better than one," the ISPSO algorithm consists of three swarms. The concrete strategies are as shown in Figure 1.

From Figure 1, it can be seen that swarm-1 and swarm-2 execute the iterative optimization separately. Swarm-3 updates the velocity by the fitness value and velocity obtaining from the above two swarms. At last, the global best value is chosen from the three fitness values.

The descending inertia weight linearly is used by the particles in swarm-1 and swarm-2 [24]. The expression of inertia weight is as shown in formula (7). The updating of velocity and position is as formula (6).

$$
w=w_{\max }-\frac{\left(w_{\max }-w_{\min }\right)}{M} t
$$

where $w_{\max }$ represents the maximum value of inertia weight, $w_{\text {min }}$ is the minimum value of inertia weight, and $M$ expresses the maximum number of iterations.

The velocity of particles in swarm-3 is updated by the fitness value and velocity obtained from swarm-1 and swarm-2. The specific expression is as shown in the following formula:

$$
\left\{\begin{array}{l}
v_{i, j}(t+1)=w\left(\frac{\lambda}{\lambda_{1}}\left(v 1_{i, j}(t)\right)+\frac{\lambda}{\lambda_{2}}\left(v 2_{i, j}(t)\right)+v_{i, j}(t)\right)+c_{1} r_{1}\left[p_{i, j}-x_{i, j}(t)\right] \\
+c_{2} r_{2}\left[p_{g, j}-x_{i, j}(t)\right] x_{i, j}(t+1)=x_{i, j}(t)+v_{i, j}(t+1),
\end{array}\right.
$$

where $\lambda_{1}$ is the fitness value of swarm-1. Similarly, $\lambda_{2}$ is the fitness value of swarm-2. $\lambda=\lambda_{1}+\lambda_{2}$ represents the sum of two fitness values. $v 1_{i, j}$ expresses the velocity of swarm-1. $v 2_{i, j}$ expresses the velocity of swarm- 2 . 
3.3. Analysis of Improved Symbiosis Particle Swarm Optimization Algorithm. In order to further explain the proposed ISPSO algorithm, the six standard test functions are used as an example $[25,26]$. Through the platform MATLAB, the convergence, robustness, and time complexity are discussed. The minimum values of six standard test functions are all zero. The expression of standard test functions is as shown in the following formula:

$$
\begin{cases}f_{\text {Sphere }}=\sum_{i=1}^{D} x_{i}^{2}, & x_{i} \in[-100,100]^{D}, \\ f_{\text {Rastrigin }}=\sum_{i=1}^{D}\left[x_{i}^{2}-10 \cos \left(2 \pi x_{i}\right)+10\right], & x_{i} \in[-10,10]^{D}, \\ f_{\text {Girewank }}=\frac{1}{4000} \sum_{i=1}^{D} x_{i}^{2}-\prod_{i=1}^{D} \cos \left(\frac{x_{i}}{\sqrt{i}}\right)+1, & x_{i} \in[-50,50]^{D}, \\ f_{\text {Schwefel }}=\sum_{i=1}^{D}\left|x_{i}\right|+\prod_{i=1}^{D}\left|x_{i}\right|, & x_{i} \in[-10,10]^{D}, \\ f_{\text {Elliptic }}=\sum_{i=1}^{D} 10^{6(i-1 / D-1)} x_{i}^{2}, & x_{i} \in[-100,100]^{D}, \\ f_{\text {Rosenbrock }}=\sum_{i=1}^{D-1}\left[100\left(x_{i}^{2}-x_{i+1}\right)^{2}+\left(x_{i}-1\right)^{2}\right], & x_{i} \in[-100,100]^{D},\end{cases}
$$

where $D$ indicates the number of independent variables.

The parameters of ISPSO algorithm are as shown in the following formula:

$$
\begin{aligned}
N & =30, \\
D & =10, \\
c_{1} & =c_{2}=2, \\
w_{\max } & =0.9, \\
w_{\text {min }} & =0.4, \\
M & =1000 .
\end{aligned}
$$

The parameters of PSO algorithm are as shown in the following formula:

$$
\begin{aligned}
& N=30, \\
& D=10, \\
& c_{1}=c_{2}=2, \\
& w=0.5, \\
& M=1000 .
\end{aligned}
$$

The parameters of ICPSO algorithm are as shown in the following formula:

$$
\begin{aligned}
N & =30, \\
D & =10, \\
c_{1} & =c_{2}=2, \\
w_{\max } & =0.9, \\
w_{\min } & =0.4, \\
\operatorname{Max} C & =10, \\
M & =1000 .
\end{aligned}
$$

The parameters of SOS algorithm are as shown in the following formula:

$$
\begin{aligned}
& N=30, \\
& D=10, \\
& M=1000 .
\end{aligned}
$$

The convergent curves of six standard test functions can be described as in Figure 2.

From Figure 2, it can be seen that the proposed ISPSO algorithm has global convergence properties for the six standard test functions. What is more, the convergence is superior to PSO algorithm, ICPSO algorithm, and SOS algorithm. In its searching later stages, the proposed ISPSO algorithm performs the fastest convergence, because of the 

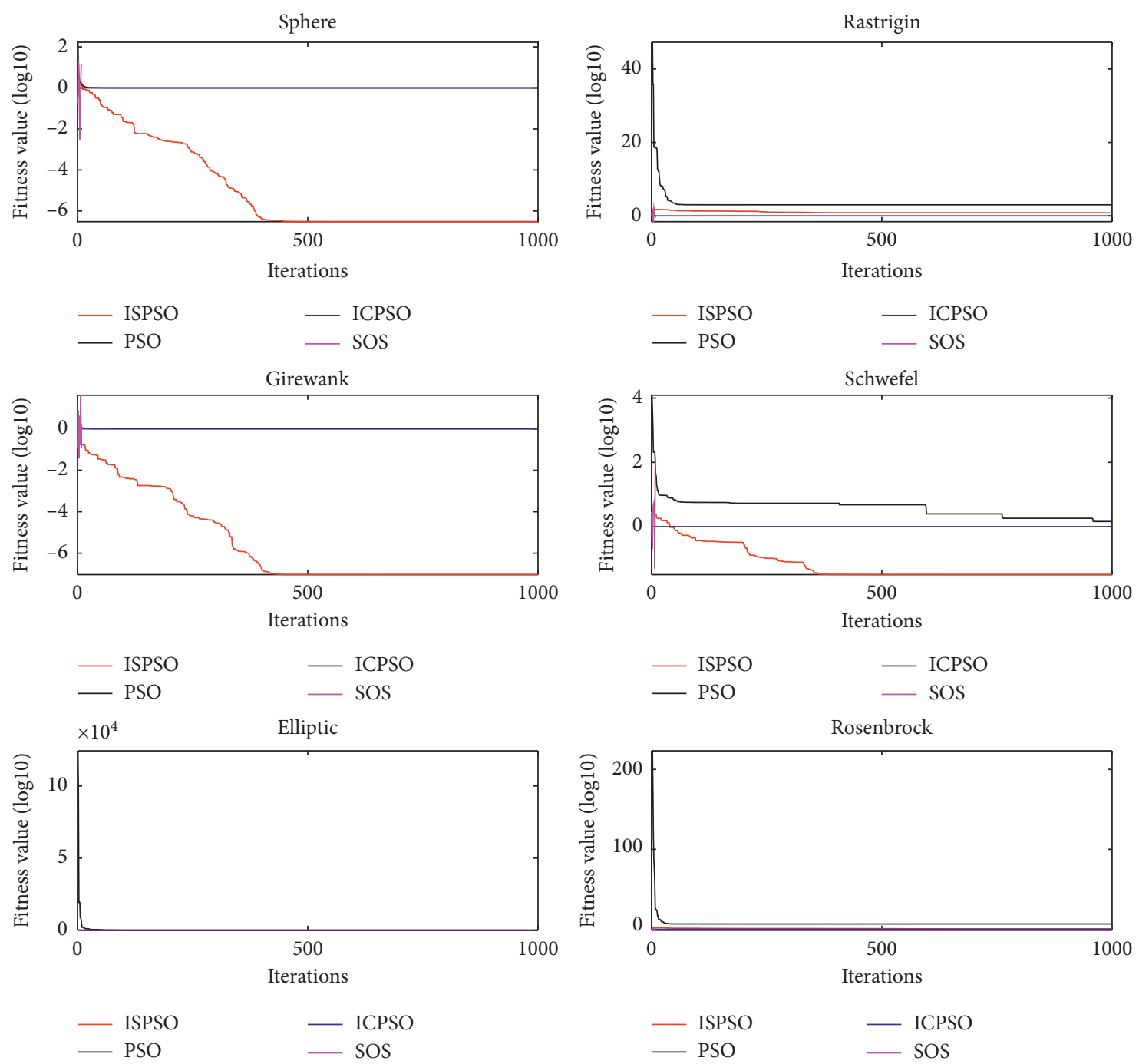

FIgURE 2: Convergent curves.

strategy of mutualistic symbiosis in nature. It improves the diversity of population. Besides, every particle performs global search well, avoiding getting into a local optimum.

In this paper, the robustness refers to the proportion reaching the threshold specified when the algorithm has the fifty operating independently. The threshold settings of six standard test functions are shown in Table 1. The calculation of success rate is as the following formula:

$$
S_{r}=\frac{50-n}{50} \times 100 \% \text {, }
$$

where $S_{r}$ is the probability of success and $n$ represents the number greater than the threshold value.

The analysis of robustness is shown in Table 2 .

From Table 2, it can be obviously seen that, for the standard test functions Sphere, Girewank, and Rosenbrock, the proposed ISPSO algorithm can be at the threshold of a hundred percent. Regarding it as a whole, compared with the PSO algorithm, the ICPSO algorithm, and the SOS algorithm, the success rate of reaching the threshold is higher by obtaining the proposed ISPSO algorithm.
In theory, the computational complexity of the proposed ISPSO algorithm, PSO algorithm, ICPSO algorithm, and SOS algorithm is as shown in the following formula:

$$
\left\{\begin{array}{l}
M_{\text {ISPSO }}=3 O(N \times D \times M) \approx O(N \times D \times M), \\
M_{\text {PSO }}=O(N \times D \times M), \\
M_{\text {ICPSO }}=O(N \times D \times M), \\
M_{\text {SOS }}=O(N \times D \times M) .
\end{array}\right.
$$

From formula (15), it can be seen that the computational complexity of the proposed ISPSO algorithm and PSO algorithm is in the same order of magnitude theoretically.

Table 3 lists the run times of six standard test functions obtained by the proposed ISPSO algorithm, PSO algorithm, ICPSO algorithm, and SOS algorithm. The calculation method uses the command statement (tic, toc) of MATLAB.

As seen in Table 3, it can be known that the run times obtained by the proposed ISPSO algorithm are in the same order, compared with the PSO algorithm, the ICPSO algorithm, and the SOS algorithm. The strategy of introduction has not increased the computational complexity of 
TABLE 1: Threshold settings of six standard test functions.

\begin{tabular}{lcccccc}
\hline Standard functions & $f_{\text {Sphere }}$ & $f_{\text {Rastrigin }}$ & $f_{\text {Girewank }}$ & $f_{\text {Schwefel }}$ & $f_{\text {Elliptic }}$ & $f_{\text {Rosenbrock }}$ \\
\hline Thresholds & $1.0 e^{-003}$ & $1.0 e^{+001}$ & $1.0 e^{-003}$ & $1.0 e^{-001}$ & $1.0 e^{+001}$ & $1.0 e^{+001}$ \\
\hline
\end{tabular}

TABLe 2: Analysis of robustness.

\begin{tabular}{lcccccc}
\hline Algorithms & $f_{\text {Sphere }}(\%)$ & $f_{\text {Rastrigin }}(\%)$ & $f_{\text {Girewank }}(\%)$ & $f_{\text {Schwefel }}(\%)$ & $f_{\text {Elliptic }}(\%)$ & $f_{\text {Rosenbrock }}(\%)$ \\
\hline ISPSO & 100 & 92 & 100 & 68 & 44 & 36 \\
PSO & 50 & 62 & 76 & 60 & 76 & 94 \\
ICPSO & 100 & 90 & 92 & 66 & 80 & 96 \\
SOS & 100 & 100 & 92 & & 98 \\
\hline
\end{tabular}

TABLE 3: Run times of two algorithms.

\begin{tabular}{lcccccc}
\hline Algorithms & $f_{\text {Sphere }}$ & $f_{\text {Rastrigin }}$ & $f_{\text {Girewank }}$ & $f_{\text {Schwefel }}$ & $f_{\text {Elliptic }}$ & $f_{\text {Rosenbrock }}$ \\
\hline ISPSO & $4 S$ & $5 S$ & $6 S$ & $6 S$ & $5 S$ & $4 S$ \\
PSO & $1 S$ & $1 S$ & $1 S$ & $1 S$ & $1 S$ & $1 S$ \\
ICPSO & $1 S$ & $1 S$ & $1 S$ & $2 S$ & $1 S$ & $3 S$ \\
SOS & $2 S$ & $2 S$ & $3 S$ & $S$ & $2 S$ \\
\hline
\end{tabular}

the algorithm, which is consistent with the theoretical analysis.

3.4. An Economic Load Dispatch Problem Based on Improved Symbiosis Particle Swarm Optimization Algorithm. The ELD problem can be solved by the proposed ISPSO algorithm, the detailed description of which is as follows:

(1) The velocity and position of all particles in the three swarms can be initialized by formula (16), which is shown as follows specifically:

$$
\left\{\begin{array}{l}
v_{i, j}=\operatorname{rand}(N, 1) *\left[\frac{\left(P_{i_{-} \max }-P_{i_{-} \min }\right)}{20}\right], \\
x_{i, j}=\operatorname{rand}(N, 1) *\left(P_{i_{-} \max }-P_{i_{-} \min }\right)+P_{i_{-} \min },
\end{array}\right.
$$

where $\operatorname{rand}(N, 1)$ represents a random number between 0 and 1 .

(2) With the above subjected functions (1) and (2), every particle calculates the objective function values by its own different iteration strategy, which is according to formula (5).

(3) The velocity and position of all particles in the three swarms can be updated through the proposed ISPSO algorithm in this paper. The particles of swarm-1 and swarm-2 are updated according to formulas (6) and (7), while the particles of swarm-3 according to formulas (7) and (8).

(4) The local optimal values outputted from every swarm are compared with each other. Then, the global optimal value is got.
The basic flowchart of ELD problem solved by the proposed ISPSO algorithm is as shown in Figure 3.

\section{Experiment and Simulation}

In order to verify the validity and efficiency of the proposed ISPSO algorithm for solving the ELD problem, the two different kinds of practical examples are used to do experiments and simulations. The practical example 3-machine-6-bus system in the literature is experimented and simulated on MATLAB, which is compared with the PSO algorithm, the ICPSO algorithm, and the SOS algorithm.

The total load power of whole system is $P=850 \mathrm{MW}$. The constraint conditions and characteristics parameters of consumption are as shown in Table 4, belonging to each generator. In the first case, the valve-point effect is not considered. On the contrary, the other case is that the valvepoint effect is considered.

4.1. Case 1. Without considering the valve-point effect, it is can be described as $e_{i}=f_{i}=0$. The remaining setting of parameters is as shown in Table 4. Because of the randomness of initialization, in order to obtain the objective and fair conclusion, each algorithm runs 50 times independently. The outputted results are analysis and statistics, which are as shown in Table 5 in detail. By experiments, the power allocation result of every generator is as displayed in Table 6.

The better results are in bold in Table 5 . From Table 5, it can be seen clearly that the best result, worst result, and average result obtained by the proposed ISPSO algorithm are all the best compared with obtaining by PSO algorithm, ICPSO algorithm, and SOS algorithm. And then, it can be concluded that the proposed ISPSO algorithm is the most effective in solving the ELD problem, which is compared 


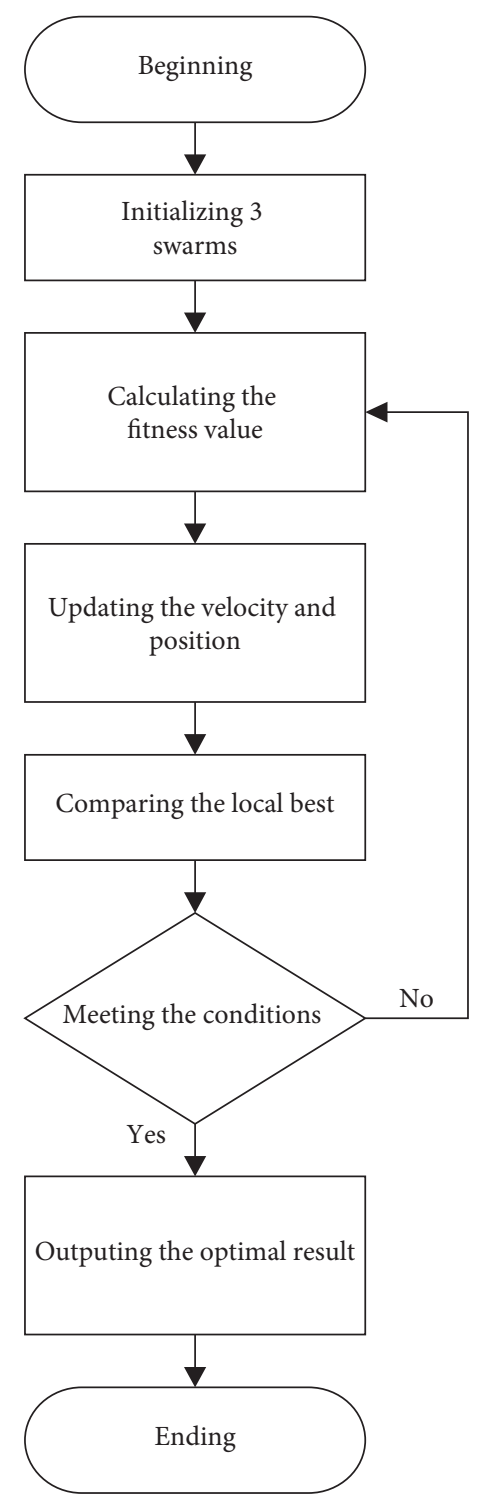

Figure 3: Basic flowchart.

TABLE 4: Constraint conditions and characteristics parameters of consumption.

\begin{tabular}{lcccccc}
\hline Generator & $P_{i_{-} \min }(\mathrm{MW})$ & $P_{i_{-} \max }(\mathrm{MW})$ & $a_{i}$ & $b_{i}$ & $c_{i}$ & $e_{i}$ \\
\hline 1 & 100 & 600 & 0.00156 & 7.92 & 561 & 300 \\
2 & 100 & 400 & 0.00194 & 7.85 & 310 & 200 \\
3 & 50 & 200 & 0.00482 & 7.97 & 78 & 0.0315 \\
\hline
\end{tabular}

TABLE 5: Outputted results without considering the valve-point effect.

\begin{tabular}{lccc}
\hline Algorithms & Best $(\$)$ & Worst $(\$)$ & Mean $(\$)$ \\
\hline ISPSO & $6.85 e^{+03}$ & $7.03 e^{+03}$ & $6.94 e^{+03}$ \\
PSO & $8.19 e^{+03}$ & $8.19 e^{+03}$ & $8.19 e^{+03}$ \\
ICPSO & $7.53 e^{+03}$ & $8.19 e^{+03}$ & $7.98 e^{+03}$ \\
SOS & $8.19 e^{+03}$ & $8.19 e^{+03}$ & $8.19 e^{+03}$ \\
\hline
\end{tabular}

TABle 6: Power allocation results without considering the valvepoint effect.

\begin{tabular}{lcccc}
\hline Power allocation & ISPSO & PSO & ICPSO & SOS \\
\hline $1(\mathrm{MW})$ & 399 & 394 & 387 & 393 \\
$2(\mathrm{MW})$ & 350 & 334 & 330 & 334 \\
$3(\mathrm{MW})$ & 101 & 122 & 133 & 123 \\
Total load power (MW) & 850 & 850 & 850 & 850 \\
Total cost (\$) & $6.93 e^{+03}$ & $8.19 e^{+03}$ & $7.69 e^{+03}$ & $8.19 e^{+03}$ \\
\hline
\end{tabular}


with the PSO algorithm, the ICPSO algorithm, and the SOS algorithm.

From Table 6, it can be seen that, compared with the PSO algorithm and the SOS algorithm, the total cost reduces $1.26 \mathrm{e}^{+03} \$$, which adopts the proposed ISPSO algorithm. Compared with the ICPSO algorithm, the total cost reduces $0.76 e^{+03} \$$ adopting the proposed ISPSO algorithm. It is further clarified that, for solving the ELD problem without considering the valve-point effect, the proposed ISPSO algorithm is more useful.

The curve of evolutionary process using the proposed ISPSO algorithm is as shown in Figure 4.

The curve of evolutionary process with the PSO algorithm is as shown in Figure 5.

The curve of evolutionary process with the ICPSO algorithm is as shown in Figure 6.

The curve of evolutionary process with the SOS algorithm is as shown in Figure 7.

The comparisons among the proposed ISPSO algorithm, PSO algorithm, ICPSO algorithm, and SOS algorithm are as shown in Figure 8.

Figure 4 shows that the evolutionary process of searching the optimal value using the proposed ISPSO algorithm. Similarly, the evolutionary process of searching the optimal value with the PSO algorithm, the ICPSO algorithm, and the SOS algorithm is as shown in Figures 5-7. Figure 8 shows the comparisons among the four algorithms. From Figures $4-8$, it can be found that the proposed ISPSO algorithm can quickly search the optimal value. What is more, it can break away from the local optimum and obtain the relatively high alignment accuracy. And so to illuminate, the proposed ISPSO algorithm in this paper is effective in solving the ELD problem without considering the valve-point effect.

4.2. Case 2. Considering the valve-point effect, the parameters set are as shown in Table 4. Due to the randomness of initialization, in order to get the objective and fair conclusion, each algorithm separately runs 50 times. The outputted results analyzed and counted are as shown in Table 7 in great detail. Through experiments, the power allocation result of every generator is as displayed in Table 8.

In Table 7, in order to directly observe, the better results are in bold. From Table 7, it can be seen that clearly the best results, worst results, and mean results got through the proposed ISPSO algorithm are superior to those got through the PSO algorithm, the ICPSO algorithm, and the SOS algorithm. It can be explained effectively that the proposed ISPSO algorithm is more suitable for solving the ELD problem considering the valve-point effect, compared with the PSO algorithm, the ICPSO algorithm, and the SOS algorithm.

From Table 8 , it can be clearly seen that the total cost reduces $1.25 e^{+03} \$$ using the proposed ISPSO algorithm, compared with the PSO algorithm. Compared with the ICPSO algorithm, it reduces $0.96 e^{+03} \$$, while, compared with the $\mathrm{SOS}$ algorithm, it reduces $1.24 e^{+03} \$$. It is further explained that, for solving the ELD problem considering the

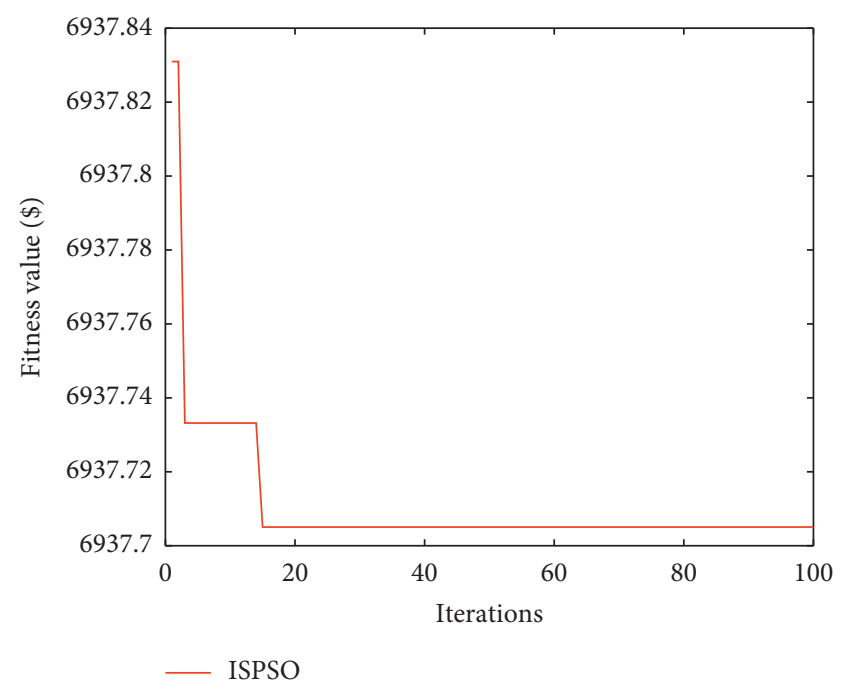

FIgURE 4: Curve of evolutionary process using the ISPSO algorithm.

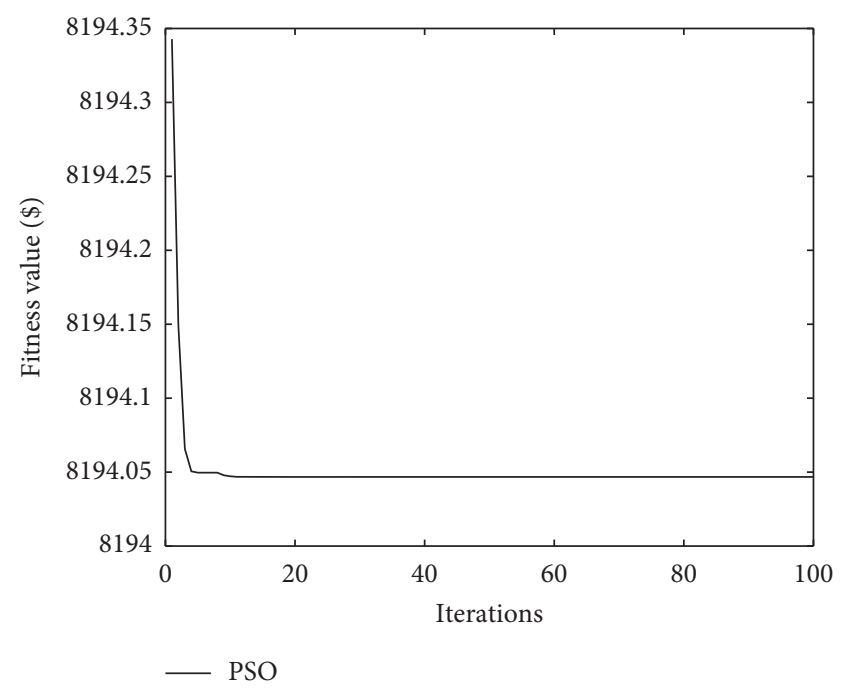

FIgURE 5: Curve of evolutionary process with the PSO algorithm.

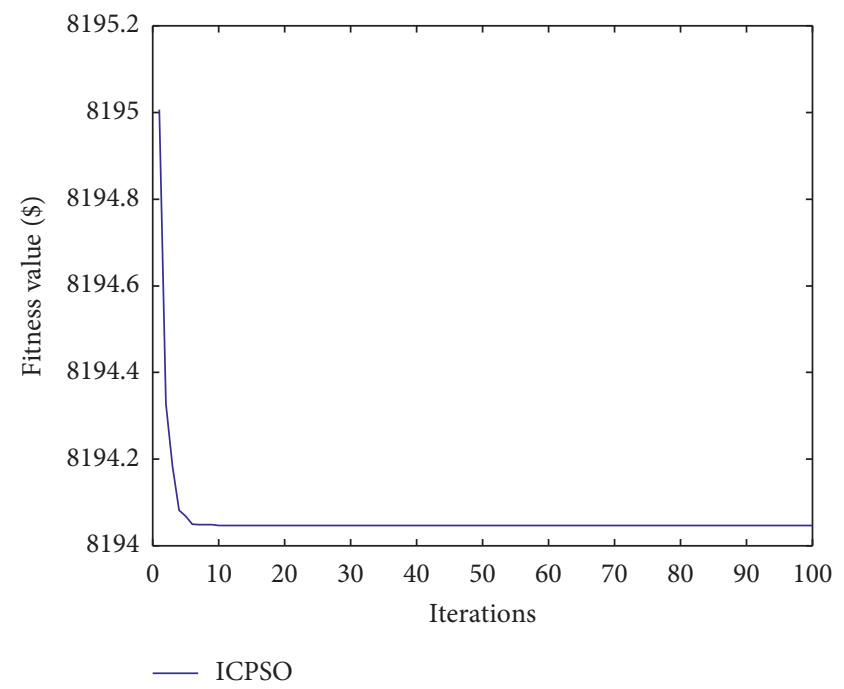

FIGURE 6: Curve of evolutionary process with the ICPSO algorithm. 


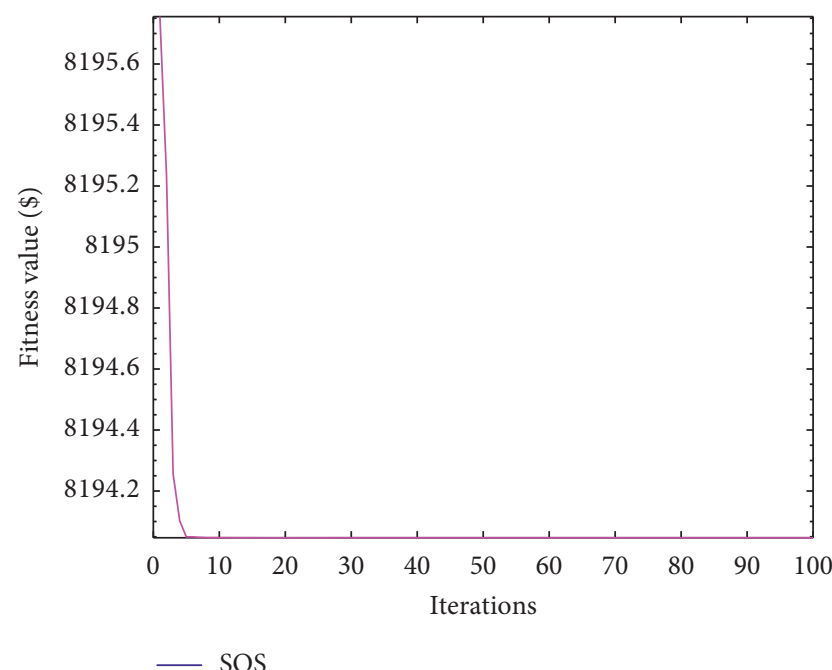

FIGURE 7: Curve of evolutionary process with the SOS algorithm.

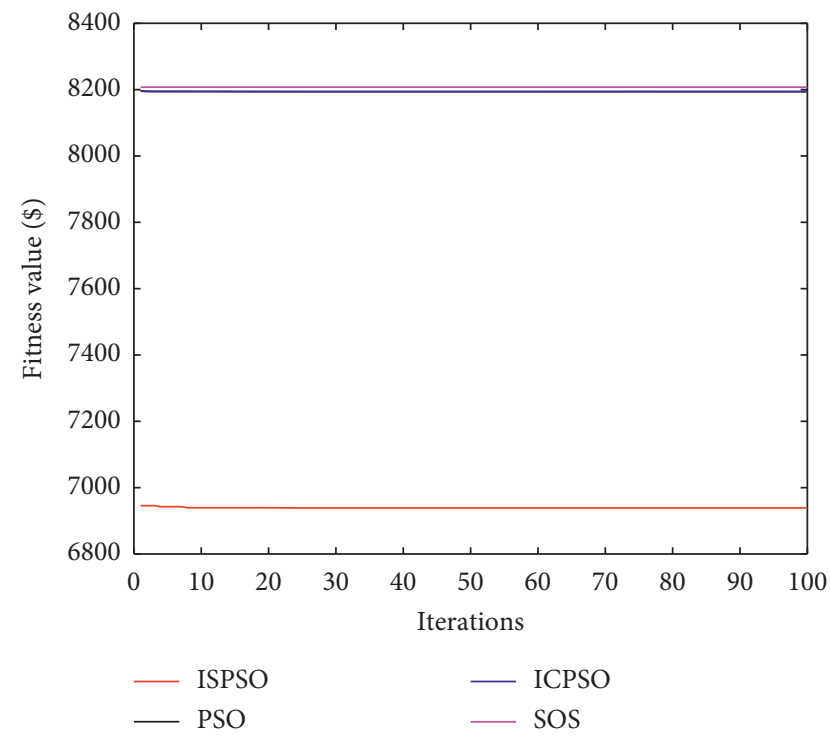

FIgURE 8: Comparisons among the four algorithms.

TABLE 7: Outputting results considering the valve-point effect.

\begin{tabular}{lccc}
\hline Algorithms & Best $(\$)$ & Worst $(\$)$ & Mean $(\$)$ \\
\hline ISPSO & $6.88 e^{+03}$ & $7.29 e^{+03}$ & $7.08 e^{+03}$ \\
PSO & $7.92 e^{+03 a}$ & $8.34 e^{+03}$ & $8.24 e^{+03}$ \\
ICPSO & $7.62 e^{+03}$ & $8.45 e^{+03}$ & $8.21 e^{+03}$ \\
SOS & $8.12 e^{+03}$ & $8.32 e^{+03}$ & $8.23 e^{+03}$ \\
\hline
\end{tabular}

TABLE 8: Power allocation results considering the valve-point effect.

\begin{tabular}{lcccc}
\hline Power allocation & ISPSO & PSO & ICPSO & SOS \\
\hline $1(\mathrm{MW})$ & 498 & 499 & 500 & 490 \\
$2(\mathrm{MW})$ & 251 & 251 & 244 & 259 \\
$3(\mathrm{MW})$ & 101 & 100 & 106 & 101 \\
Total load power (MW) & 850 & 850 & 850 & 850 \\
Total cost (\$) & $6.99 e^{+03}$ & $8.24 e^{+03}$ & $7.95 e^{+03}$ & $8.23 e^{+03}$ \\
\hline
\end{tabular}

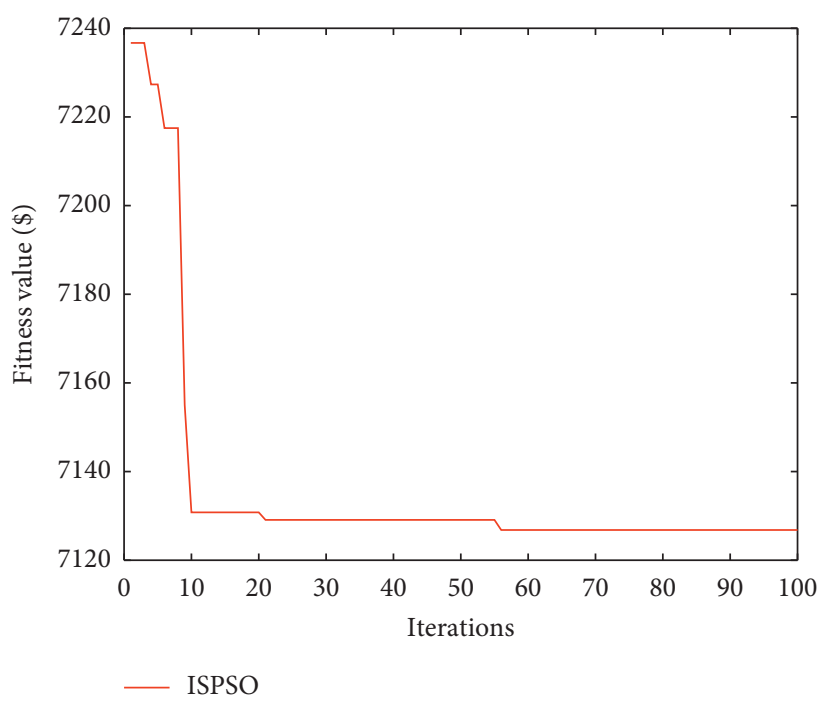

FIGURE 9: Curve of evolutionary process through the ISPSO algorithm.

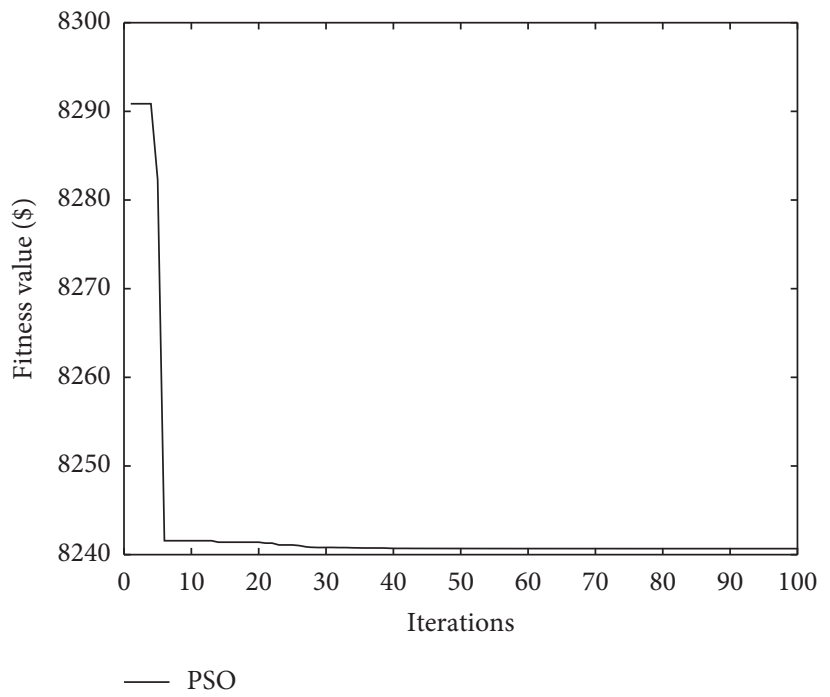

FIGURE 10: Curve of evolutionary process with the PSO algorithm.

valve-point effect, the proposed ISPSO algorithm is the most effective.

The curve of evolutionary process obtained through the proposed ISPSO algorithm is as shown in Figure 9.

The curve of evolutionary process got by the PSO algorithm is as shown in Figure 10.

The curve of evolutionary process got by the ICPSO algorithm is as shown in Figure 11.

The curve of evolutionary process got by the SOS algorithm is as shown in Figure 12.

The comparisons among the four algorithms are as shown in Figure 13.

The evolutionary process of searching the optimal value through the proposed ISPSO is as shown in Figure 9. Also, Figures $10-12$, respectively, show the evolutionary process of searching the optimal value with the PSO algorithm, the ICPSO algorithm, and the SOS algorithm. Figure 13 shows 


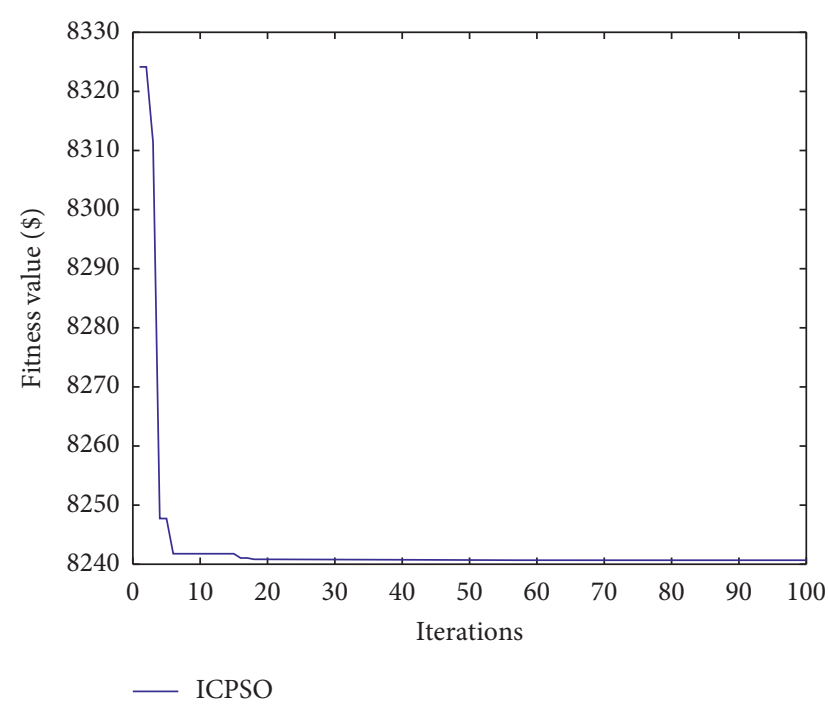

FIGURE 11: Curve of evolutionary process with the ICPSO algorithm.

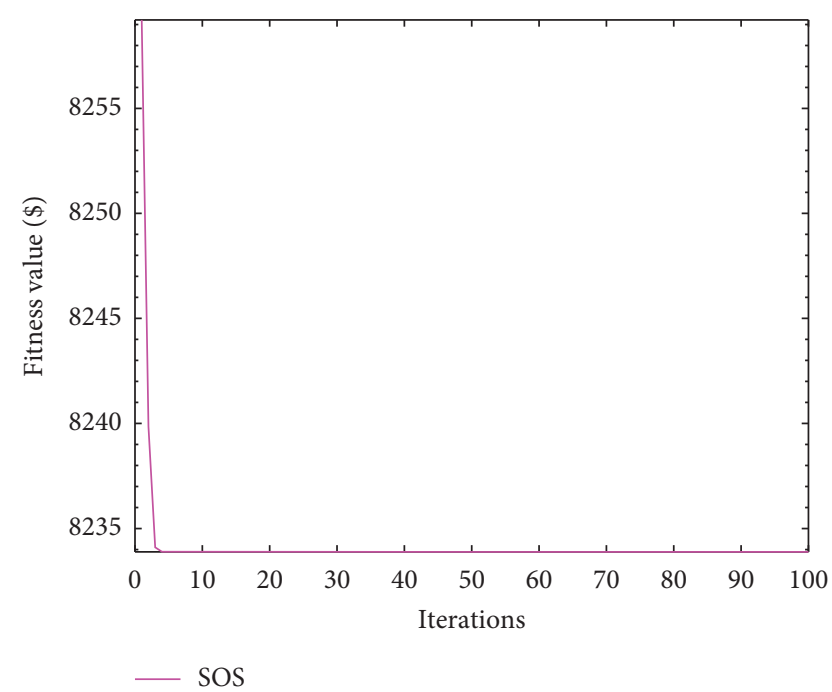

FIGURE 12: Curve of evolutionary process with the SOS algorithm.

the comparison among the four algorithms. From Figure 13, it can be observed that, for solving the ELD problem considering the valve-point effect, the proposed ISPSO algorithm in the convergence rate and the ability to jump out to local optimum algorithm is the best compared with the PSO algorithm, the ICPSO algorithm, and the SOS algorithm. It can be absolutely ensured that the proposed ISPSO algorithm is the best in solving the ELD problem considering the valve-point effect.

\section{Conclusions}

In this paper, an ISPSO algorithm was proposed for solving the ELD problem. The ISPSO algorithm successfully introduced the strategy of mutualistic symbiosis in nature, which was that biological populations would benefit and influence each other. Besides, the ISPSO algorithm adopted three swarms inspired by

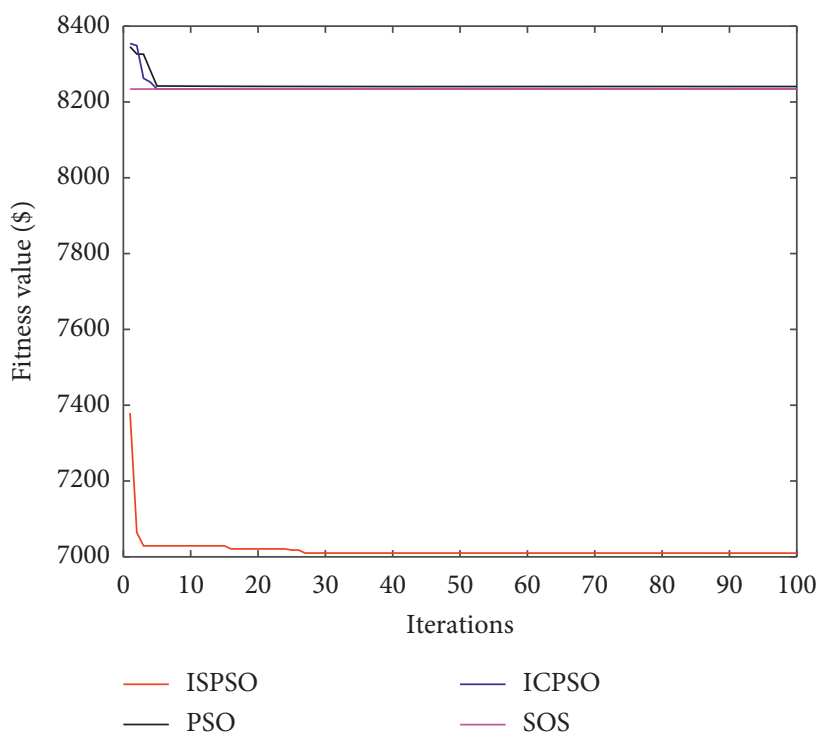

FIGURE 13: Comparisons among the four algorithms.

the proverb "two heads are better than one," where swarm-1 and swarm-2 carried on iteration optimization, and swarm-3 utilized the position information of the above two swarms to self-update. Moreover, the descending inertia weight linearly is utilized by the particles to improve the defects of precocious convergence. To solve the ELD problem well, it was converted into a mathematical model using certain conditions in accordance with the reality. Then, the specific analysis of ISPSO was through the standard test functions, and two different kinds of practical examples were employed for the evaluation experiments. The compared simulation results with PSO algorithm, ICPSO algorithm, and SOS algorithm illustrated the effectiveness of the proposed ISPSO algorithm in solving the ELD problem.

\section{Data Availability}

Two different kinds of practical examples are used to perform experiments and simulations, which can be downloaded from https://xueshu.baidu.com/usercenter/paper/ show?paperid=bee6f4bdf62a1e9963f9512f59738d45

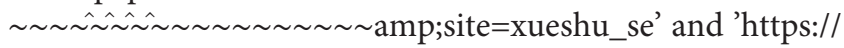
kns.cnki.net/KXReader/Detail?TIMESTAMP $=63735014615$

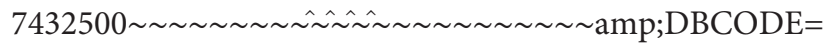

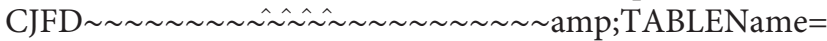

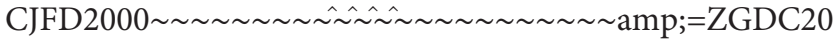

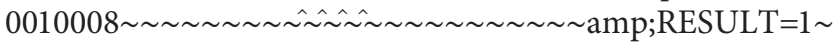

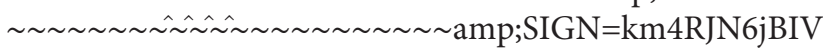

O6W7fXoDzoMfKgFU\%3d.

\section{Additional Points}

Highlights. An improved symbiosis particle swarm optimization algorithm is proposed for economic load dispatch problem. The improved optimization algorithm consists of three swarms inspired by the proverb and nature. The given optimization algorithm is extensively evaluated through the standard test functions and economic load dispatch 
problem. Compared experiment results on convergence and stability analyses demonstrate the effectiveness of the improved algorithm.

\section{Conflicts of Interest}

The authors declare that they have no conflicts of interest.

\section{Acknowledgments}

This research was supported financially by Henan Provincial Department of Science and Technology Research Project (grant nos. 202102210168 and 192102110198 ), Key Scientific Research Projects of Colleges and Universities in Henan Province (grant no. 20B590001), and Henan Institute of Technology Doctoral Research Fund Project (grant no. KQ1812).

\section{References}

[1] J. B. Park, Y. W. Jeong, and J. R. Shin, “An improved particle swarm optimization for nonconvex economic dispatch problems," IEEE Transactions on Power Systems, vol. 25, no. 1, pp. 156-166, 2020.

[2] M. S. P. Subathra, S. E. Selvan, T. A. A. Victoire, A. H. Christinal, and U. Amato, "A hybrid with cross-entropy method and sequential quadratic programming to solve economic load dispatch problem," IEEE Systems Journal, vol. 9, no. 3, pp. 1031-1044, 2015.

[3] S. Kar, G. Hug, J. Mohammadi, and J. M. F. Moura, "Distributed state estimation and energy management in smart grids: a Consensus $\$\{+\}$ Innovations approach," IEEE Journal of Selected Topics in Signal Processing, vol. 8, no. 6, pp. 1022-1038, 2014.

[4] M. Fesanghary and M. M. Ardehali, "A novel meta-heuristic optimization methodology for solving various types of economic dispatch problem," Energy, vol. 34, no. 6, pp. 757-766, 2009.

[5] J. S. Alsumait, J. K. Sykulski, and A. K. Al-Othman, "A hybrid GA-PS-SQP method to solve power system valve-point economic dispatch problems," Applied Energy, vol. 87, no. 5, pp. 1773-1781, 2010.

[6] B. R. Adarsh, T. Raghunathan, T. Jayabarathi, and X.-S. Yang, "Economic dispatch using chaotic bat algorithm," Energy, vol. 96, pp. 666-675, 2016.

[7] X. He, Y. Rao, and J. Huang, "A novel algorithm for economic load dispatch of power systems," Neurocomputing, vol. 171, pp. 1454-1461, 2016.

[8] L. Han, C. E. Romero, and Z. Yao, "Economic dispatch optimization algorithm based on particle diffusion," Energy Conversion and Management, vol. 105, pp. 1251-1260, 2015.

[9] J. S. Al-Sumait, A. K. Al-Othman, and J. K. Sykulski, “Application of pattern search method to power system valvepoint economic load dispatch," International Journal of Electrical Power \& Energy Systems, vol. 29, no. 10, pp. 720730, 2007.

[10] C. Cheng, S. Li, and G. Li, "A hybrid method of incorporating extended priority list into equal incremental principle for energy-saving generation dispatch of thermal power systems," Energy, vol. 64, pp. 688-696, 2014.

[11] J. X. Xu, T. Sicong, and S. K. Panda, "Optimization of economic load dispatch for a micro-grid using evolutionary computation," in Proceedings of the IECON 2011-37th Annual
Conference of the IEEE Industrial Electronics Society, pp. 192-197, IEEE, Melbourne, Australia, November 2011.

[12] S. H. Ling, H. K. Lam, and F. H. F. Leung, "Improved genetic algorithm for economic load dispatch with valve-point loadings," in Proceedings of the 29th Annual Conference of the IEEE Industrial Electronics Society, pp. 442-447, IEEE, Roanoke, VA, USA, USA, November2003.

[13] X. B. Li, "Study of multi-objective optimization and multiattribute decision marking of economic load dispatch problem," Proceeding of the CSEE, vol. 28, no. 35, pp. 102-107, 2008.

[14] W. L. Xiong, M. Xu, and B. G. Xu, "Differential bee colony algorithm for non-convex load dispatch," Control and Decision, vol. 12, no. 12, pp. 1813-1817, 2011.

[15] C. B. Han, X. M. Lv, F. Q. Si et al., "Study on economic load dispatch based on improved CPSO algorithm for thermal power plants," Journal of Chinese Society of Power Engineering, vol. 35, no. 4, pp. 312-317, 2015.

[16] R. P. Parouha and K. N. Das, "A novel hybrid optimizer for solving Economic Load Dispatch problem," International Journal of Electrical Power \& Energy Systems, vol. 78, pp. 108-126, 2016.

[17] T. Sen and H. D. Mathur, "A new approach to solve Economic Dispatch problem using a Hybrid ACO-ABC-HS optimization algorithm," International Journal of Electrical Power \& Energy Systems, vol. 78, no. 78, pp. 735-744, 2016.

[18] M. Gholamghasemi, E. Akbari, M. B. Asadpoor, and M. Ghasemi, "A new solution to the non-convex economic load dispatch problems using phasor particle swarm optimization," Applied Soft Computing, vol. 79, pp. 111-124, 2019.

[19] A. Faramarzi, M. Heidarinejad, S. Mirjalili et al., "Marine Predators algorithm: a nature-inspired metaheuristic," Expert Systems with Applications, vol. 152, pp. 1-28, 2020.

[20] A. M. Schaheen, A. M. Elsayed, R. A. El-Sehiemy et al., "Equilibrium optimization algorithm for network reconfiguration and distributed generation allocation in power systems," Applied Soft Computing Journal, vol. 98, 2020.

[21] A. M. Schaheen and R. A. El-Sehiemy, "A multiobjective salp optimization algorithm for techno-economic-based performance enhancement," IEEE Systems Journal, 2020.

[22] I. J. Balaguer, Q. Lei, S. Yang, U. Supatti, and F. Z. Peng, "Control for grid-connected and intentional islanding operations of distributed power generation," IEEE Transactions on Industrial Electronics, vol. 58, no. 1, pp. 147-157, 2011.

[23] R. Eberhat and J. Kennedy, "Particle swarm optimization," IEEE International Conference on Neural Networks, vol. 11-12, pp. 1942-1948, 1995.

[24] J. Zhang and X. Ding, "A multi-swarm self-adaptive and cooperative particle swarm optimization," Engineering Applications of Artificial Intelligence, vol. 24, no. 6, pp. 958-967, 2011.

[25] K. T. Li, N. O. Mohammad, Z. Y. Yang et al., "Benchmark functions for the CEC' 2013 special session and competition on large-scale global optimization," IEEE Congress on Evolutionary Computation, 2013.

[26] T. Wei and D. P. Li, "Chaotic optimization for economic dispatch of power systems," Proceedings of the CSEE, vol. 20, no. 10 , pp. $36-40,2000$. 